\title{
SRSF7 knockdown promotes apoptosis of colon and lung cancer cells
}

\author{
YU FU and YINGZE WANG \\ College of Bioscience and Bioengineering, Hebei University of Science and Technology, \\ Shijiazhuang, Hebei 050018, P.R. China
}

Received June 14, 2017; Accepted January 10, 2018

DOI: $10.3892 / \mathrm{ol} .2018 .8072$

\begin{abstract}
Serine/arginine-rich (SR) proteins are a family of important splicing factors, which are involved in multiple aspects of RNA processing, including splicing, mRNA nuclear export, mRNA stability and translation. Previous studies have identified a number of SR proteins that exhibit abnormal expression in various tumor types. In the present study, the expression and function of serine/arginine-rich splicing factor 7 (SRSF7) were investigated in colon and lung cancer. Using tissue immunohistochemistry, it was observed that SRSF7 was overexpressed in colon and lung cancer tissues. As the role of SRSF7 in cancer remains to be fully elucidated, the expression of SRSF7 was knocked down in the present study by transfecting SRSF7-specific small interfering RNAs (siRNAs) into the HCT116 colon cancer cell line and A549 lung cancer cell line, which exhibited elevated expression of SRSF7. MTS assays, western blot analysis, flow cytometry and spectrofluorometer analyses were performed to assess the effects of SRSF7 knockdown on the proliferation and apoptosis of cells. The results demonstrated that the expression of SRSF7 was efficiently knocked down by SRSF7 siRNA, and that SRSF7 knockdown inhibited proliferation and enhanced apoptosis of HCT116 and A549 cells. Further experiments involving BEAS-2B cells stably overexpressing SRSF7, and A549 cells with stable knockdown of SRSF7 revealed that SRSF7 regulated the splicing of the apoptosis regulator Fas. Collectively, these data indicated that SRSF7 is critical for the survival of colon and lung cancer cells, and may be a potential therapeutic target for the treatment of colon and lung cancer.
\end{abstract}

Correspondence to: $\mathrm{Dr} \mathrm{Yu} \mathrm{Fu}$, College of Bioscience and Bioengineering, Hebei University of Science and Technology, 26 Yuxiang Street, Shijiazhuang, Hebei 050018, P.R. China E-mail: fuyu@hebust.edu.cn

Key words: serine/arginine-rich splicing factor 7, cell apoptosis, colon cancer, lung cancer, splicing

\section{Introduction}

Serine/arginine-rich (SR) proteins are a conserved RNA-binding protein family, which consists of 12 members, serine/arginine-rich splicing factor (SRSF)1-12, in humans $(1,2)$. SR proteins have multiple key roles in the control of gene expression, including constitutive and alternative pre-mRNA splicing, transcription, mRNA transport, mRNA stability and translation (3-5). Changes in the expression of SR proteins may lead to aberrant alternative splicing and potentially contribute to various diseases, and in particular, to the development of cancer. Previous studies have indicated that the expression of the majority of SR proteins is altered in various tumor types, and several SR proteins, acting as proto-oncogenes, are frequently upregulated in cancer (6-8).

SRSF7, previously known as 9G8, is a member of the SR protein family that was identified in $1994(9,10)$. SRSF7 comprises an RNA-recognition motif at the N-terminus that provides RNA-binding specificity, an arginine/serine domain at the $\mathrm{C}$-terminus that promotes protein-protein interactions to facilitate spliceosome assembly, and a zinc-knuckle domain, which is thought to contact the RNA. SRSF7 regulates the constitutive splicing and alternative splicing of various pre-mRNAs, including CD44 (11), CD45 (12), BRCA1 (13) and Tau (14). Furthermore, SRSF7 can shuttle continuously between the nucleus and cytoplasm, with additional involvement in mRNA transport and translation $(15,16)$. Several studies have suggested that SRSF7 may have an active role in cancer cells $(13,17,18)$. However, the precise effects of SRSF7 on cancer cells remain to be elucidated.

The Fas protein, also known as CD95, is a widely expressed cell-surface death receptor, which, upon binding to Fas ligand, can initiate a cascade that eventually leads to programmed cell death $(19,20)$. The alternative splicing of Fas receptor pre-mRNA may be an important strategy used by tumors cells to evade elimination by the immune system. Fas exon 6 encodes a transmembrane domain, and skipping of this exon produces an mRNA encoding a soluble Fas isoform, which prevents cell death $(21,22)$. Increased levels of the soluble isoform of Fas have been identified in several types of cancer (23-25). Tejedor et al (26) demonstrated that iron homeostasis affects the alternative splicing of Fas receptor pre-mRNA by SRSF7. However, little is known about SRSF7 and Fas splicing in cancer cells. 
In the present study, it was demonstrated that SRSF7 proteins were expressed at high levels in colon and lung cancer, both of which have increasing rates of incidence and mortality worldwide $(27,28)$. In addition, it was found that SRSF7 knockdown inhibited proliferation and enhanced apoptosis of colon and lung cancer cells. Finally, it was found that SRSF7 targeted the apoptosis regulator Fas in cancer cells, which may explain a number of the activities of SRSF7.

\section{Materials and methods}

Cell culture. The HCT116 cells were cultured in McCoy's 5A medium (M\&C Gene Technology Ltd., Beijing, China). The A549, H1975, H1299 and NCM460 cells were cultured in RPMI-1640 (M\&C Gene Technology Ltd.). The BEAS-2B, HCoEpic and SW620 cells were cultured in DMEM (M\&C Gene Technology Ltd.). All culture media were supplemented with $10 \%$ fetal bovine serum (Gibco, Thermo Fisher Scientific, Inc., Waltham, MA, USA), penicillin and streptomycin. All the cells were obtained from the Cell Bank of Chinese Academy of Sciences (Shanghai, China) and incubated in a humidified atmosphere of $5 \% \mathrm{CO}_{2}$ at $37^{\circ} \mathrm{C}$.

Transfection and RNA interference. Small interfering RNA (siRNA) transfections were performed using Lipofectamine 2000 (Invitrogen; Thermo Fisher Scientific, Inc.), according to the manufacturer's protocol. siRNA synthesis was performed by Shanghai GenePharma Co., Ltd. (Shanghai, China) and the siRNA sequences for human SRSF7 were as follows: SRSF7-1, 5'-AGGAGAGUUAGAAAGGGCU-3'; and SRSF7-2, 5'-GCA UCUCCUCGACGAUCAA-3'. The sequence of the control siRNA was 5'-UUCUCCGAACGUGUCACGUTT-3'.

Western blot, reverse transcription-polymerase chain reaction (RT-PCR) and MTS cell proliferation analyses. The methods were performed as described previously (29). For western blot analysis, cells were lysed with radioimmunoprecipitation assay cell lysis buffer (Beijing Solarbio Science \& Technology Co., Ltd., Beijing, China) containing $1 \mathrm{mM}$ phenylmethylsulfonyl fluoride and quantified using a bicinchoninic acid assay Kit (Beijing Solarbio Science \& Technology Co., Ltd.). Equal amounts of protein $(30 \mu \mathrm{g})$ were separated via SDS-PAGE (12\% gel) and then transferred to polyvinylidene difluoride membranes (EMD Millipore, Billerica, MA, USA). The membrane was blocked for $1 \mathrm{~h}$ with $5 \%$ skimmed milk at room temperature and then incubated with primary antibodies overnight at $4^{\circ} \mathrm{C}$. The primary antibody against SRSF7 (AP12306a, 1:500) was purchased from Abgent, Inc. (San Diego, CA, USA). The primary antibodies against $\beta$-tubulin (10068-1-AP, $1: 1,000)$ and $\beta$-actin $(60008-1-I g$. 1:3,000) was purchased from Proteintech Group, Inc. (Rosemont, PA, USA). Following three washes in Tris-buffered saline with Tween-20, the membrane was incubated with secondary goat anti-rabbit (SA00001-2) or goat anti-mouse (SA00001-1) antibody (Proteintech Group, Inc) for $1 \mathrm{~h}$ at $37^{\circ} \mathrm{C}$ with a dilution of $1: 3,000$. Finally, the proteins were visualized using EasySee Western Blot Kit (Beijing TransGen Biotech Co., Ltd., Beijing, China), imaged and quantified using ChemiDoc MP Imaging System (Image Lab Software, version 4.1; Bio-Rad Laboratories Co., Ltd., Hercules, CA, USA).
For RT-PCR, total RNA was extracted using TRIzol reagent (Life Technologies; Thermo Fisher Scientific, Inc.), and reverse transcription was performed using a Reverse Transcription system (Promega Corporation, Fitchburg, WI, USA). Total RNA ( $4 \mu \mathrm{g}$ ) was reverse transcribed using TransScript One-Step gDNA Removal and cDNA Synthesis SuperMix (Beijing TransGen Biotech Co., Ltd.) according to the manufacturer's protocol. The thermocycling protocol was listed as follows: Initial denaturation at $95^{\circ} \mathrm{C}$ for $5 \mathrm{~min}$, followed by 30 repeats of the threestep cycling program consisting of $30 \mathrm{sec}$ at $95^{\circ} \mathrm{C}$ (denaturation), $30 \mathrm{sec}$ at $60^{\circ} \mathrm{C}$ (primer annealing) and $30 \mathrm{sec}$ at $72^{\circ} \mathrm{C}$ (elongation), followed by a final extension step for $5 \mathrm{~min}$ at $72^{\circ} \mathrm{C}$. The primers used were as follows: SRSF7, forward 3'-GCGGTACGG AGGAGAAAC-5' and reverse 3'-TCGGGAGCCACAAAT CAC-5'; Fas, forward 3'-GAACATGGAATCATCAAGGAA TGCAC-5' and reverse 3'-AGTTGGAGATTCATGAGA ACCTTGG-5'. The primers used to detect the alternative splicing of Fas were as follows: FAS-L, forward 3'-TGC AAAGAGGAAGGATCCAG-5'; FAS-S, forward 3'-CCA AGTGCAAAGAGGAAGTGA-5'; and FAS-L and FAS-S reverse 3'-GGAGAT TCATGAGAACCTTGG-5' (26). The housekeeping gene GAPDH was used as the internal control.

Analysis of cell apoptosis. The HCT116 and A549 cells were transfected with control siRNA or SRSF7 siRNA for 60-72 h. Following transfection, $1 \times 10^{6}$ cells were harvested, washed twice in PBS and double-stained with Annexin V-FITC and PI using an Annexin V-FITC/PI Cell Apoptosis Detection kit (Beijing TransGen Biotech Co.) according to the manufacturer's protocol. Each sample was then quantitatively analyzed with an Accuri C6 flow cytometer (BD Biosciences, San Jose, CA, USA) at $488 \mathrm{~nm}$ emission and $570 \mathrm{~nm}$ excitation. Cell apoptosis was also examined using an Apo-ONE Homogeneous Caspase-3/7 assay (Promega Corporation) according to the manufacturer's protocol. Caspase substrate and buffer from the kit were added for cell lysis and incubated at room temperature for $18 \mathrm{~h}$. Fluorescence was measured with an F-7000 spectrofluorometer (Hitachi, Ltd., Tokyo, Japan) at $521 \mathrm{~nm}$ emission and $499 \mathrm{~nm}$ excitation wavelengths.

Generation of stable cell lines. The BEAS-2B cells were infected with a lentivirus LV5-negative control vector or a LV5-SRSF7 vector, which were purchased from Shanghai GenePharma Co., Ltd. The A549 cells were infected with lentivirus LV3-negative control, LV3-SRSF7 short hairpin shRNA-1 (3'-GATCAAGATCCAGGTCTATTT-5') or LV3-SRSF7 shRNA-2 (3'-GAACTGTATGGATTGCGA GAA-5'), which were purchased from Shanghai GenePharma Co., Ltd. At 48 h post-infection, the cells were cultured in the aforementioned medium containing puromycin $(0.5 \mu \mathrm{g} / \mathrm{ml})$, and the medium was replenished every 2 days. After $\sim 1$ week, stable cell lines were obtained and verified by western blot analysis, as described above.

Immunohistochemical analysis. Immunohistochemistry was performed by Cybrdi, Inc. (Xi'an, China) on a multi-organ (colon, pancreas, lung, breast and prostate) tumor and normal tissue array (cat. no. MC1801, 26 spots for each tumor 
A
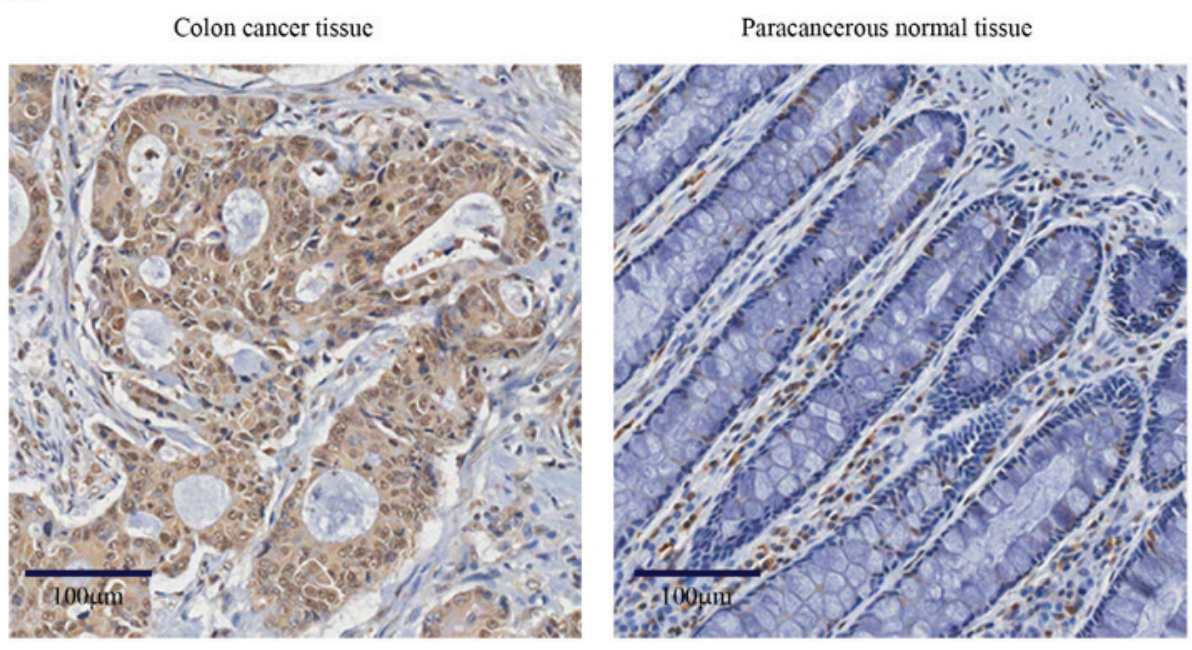

$\mathrm{B}$
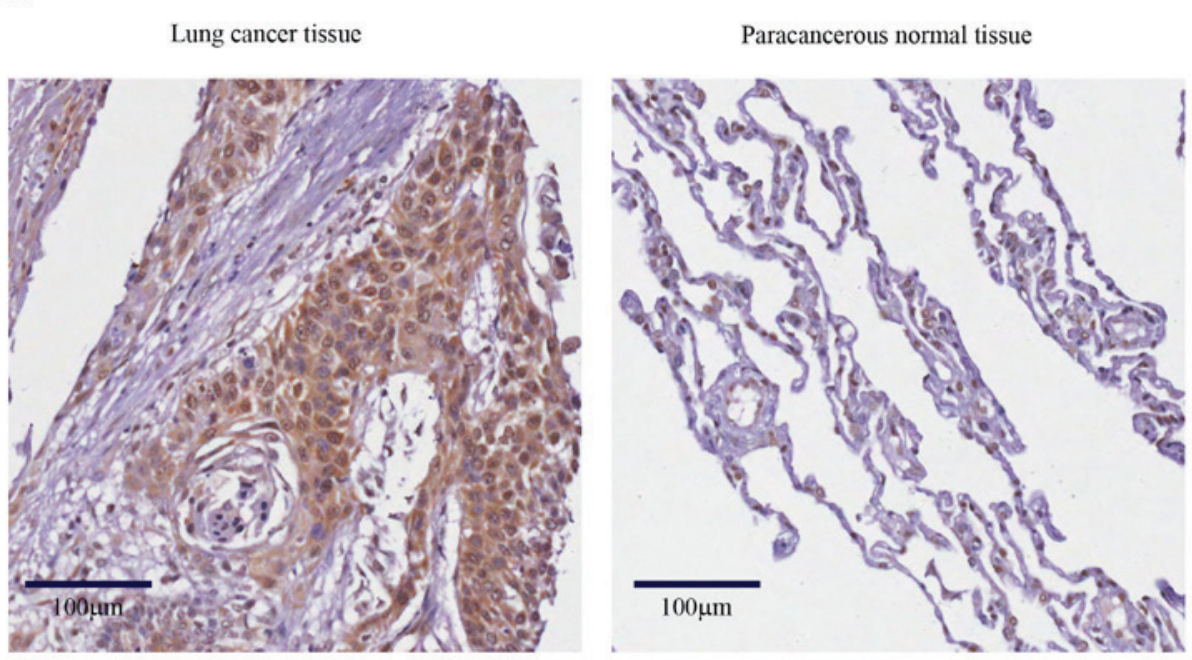

Figure 1. SRSF7 is overexpressed in human colon and lung cancer. (A) Representative immunohistochemical images of the expression of SRSF7 in human colon cancer samples and paracancerous normal tissue. Scale bar, $100 \mu \mathrm{m}$. (B) Representative immunohistochemical images of the expression of SRSF7 in human lung cancer samples and paracancerous normal tissue. Scale bar, $100 \mu \mathrm{m}$. SRSF7, serine/arginine-rich splicing factor 7.

type), human colon carcinoma (grades I-IV) tissue array (cat.no. CO1801,90 spots for cancer tissues and paracancerous normal tissues, respectively), and human lung carcinoma (grades I-III) tissue array (cat. no. LC10012a, 45 spots for cancer tissues and paracancerous normal tissues, respectively). The absent or abnormal tissues were not finally counted. Anti-SRSF7 (Abgent, Inc.) antibodies were used at a dilution of 1:50 (for array MC1801), 1:150 (for array CO1801) and 1:100 (for array LC10012a) at $4^{\circ} \mathrm{C}$ for overnight. Semi-quantitative analysis of the stained sections (H-score) was performed with a light microscope (Model, CX31; Olympus, Tokyo, Japan) by an independent pathologist.

Statistical analysis. All statistical analyses were performed by two-tailed Student's t-test or a one-way analysis of variance followed by Bonferroni's post-hoc test. Data are presented as the mean \pm standard deviation. $\mathrm{P}<0.05$ was considered to indicate a statistically significant difference. Computer-based calculations were conducted using SPSS 19.0 software (IBM Corp., Armonk, NY, USA).

\section{Results}

SRSF7 is upregulated in colon and lung cancer. To evaluate the role of SRSF7 in human cancer, its expression was analyzed on cancer arrays with different tissue origins by immunohistochemical staining. When compared with corresponding normal tissues, a high frequency of increased expression of SRSF7 was found in colon adenocarcinoma (21/24) and lung carcinoma (22/24). This result was confirmed with the specific human colon and lung cancer specimens. As shown in Fig. 1A and B, the immunostaining intensity of SRSF7, compared with paracancerous normal tissues, was more marked in the colon cancer samples (49/71) and lung cancer samples (28/39). These results suggested that SRSF7 offers potential as a marker for colon and lung cancer development, and may be involved in the development of these types of cancer.

Downregulation of SRSF7 inhibits the proliferation of HCT116 and A549 cells. The expression of SRSF7 was analyzed 
A
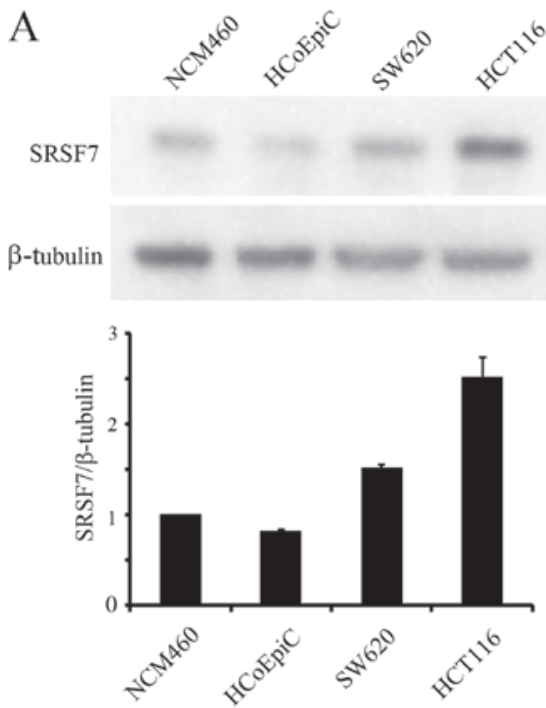

$\mathrm{C}$
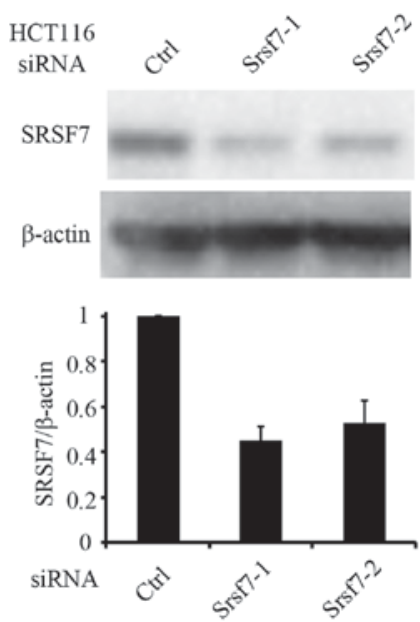

B
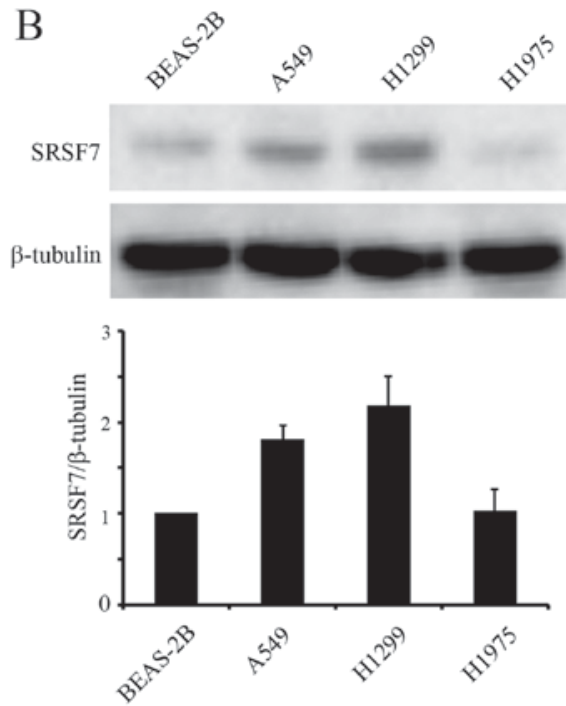

$\mathrm{D}$
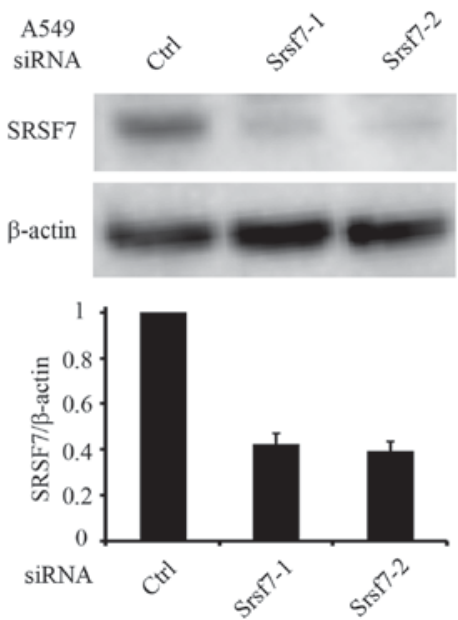

Figure 2. Protein expression of SRSF7 in human colon and lung cells. (A) Western blot analysis of the expression of SRSF7 and $\beta$-tubulin in human normal colon epithelial cells (NCM460 and HoEpiC) and human colon cancer cells (HCT116 and SW620). The relative expression of SRSF7 against $\beta$-tubulin was determined from three independent experiments. (B) Western blot analysis of the expression of SRSF7 and $\beta$-tubulin in human normal lung epithelial cells (BEAS-2B) and human lung cancer cells (A549, H1299 and H1975). The relative expression of SRSF7 against $\beta$-tubulin was determined from three independent experiments. Western blot analysis of the expression of SRSF7 and $\beta$-actin in (C) HCT116 and (D) A549 cells transfected with SRSF7 siRNA or control siRNA. Proteins were extracted at $48 \mathrm{~h}$ post-transfection. The relative expression of SRSF7 against $\beta$-actin was quantified with three independent experiments. SRSF7, serine/arginine-rich splicing factor 7; siRNA, small interfering RNA; Ctrl, control.
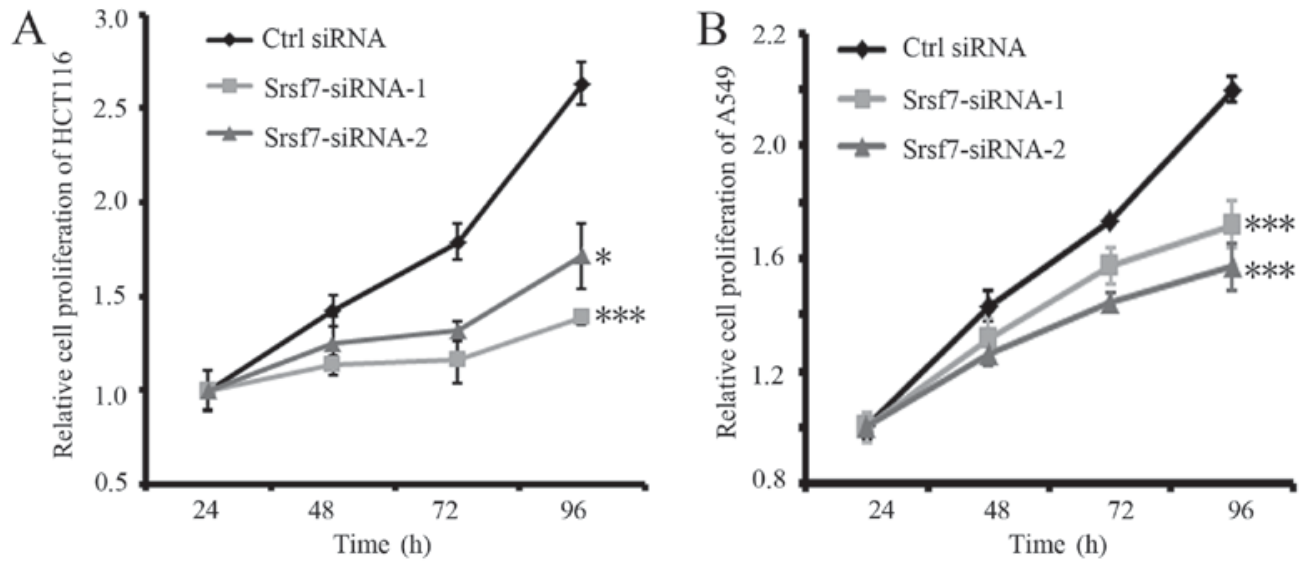

Figure 3. Downregulation of SRSF7 inhibits cell proliferation in colon and lung cancer cells. (A and B) Results of an MTS cell proliferation assay showing that the knockdown of SRSF7 in (A) HCT116 and (B) A549 cells reduced cell proliferation. The OD value at $490 \mathrm{~nm}$ at $24 \mathrm{~h}$ was defined as 1 . Results are representative of at least three independent replicates, and error bars represent the standard deviation. ${ }^{*} \mathrm{P}<0.05$ and ${ }^{* * *} \mathrm{P}<0.001$, vs. Ctrl at 96 h. SRSF7, serine/arginine-rich splicing factor 7; Ctrl, control. 


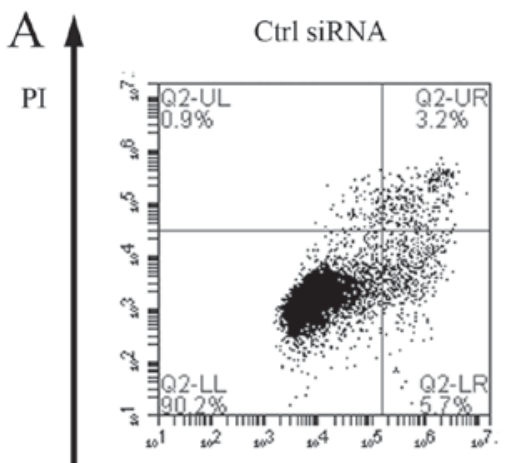

Srsf7-siRNA-1

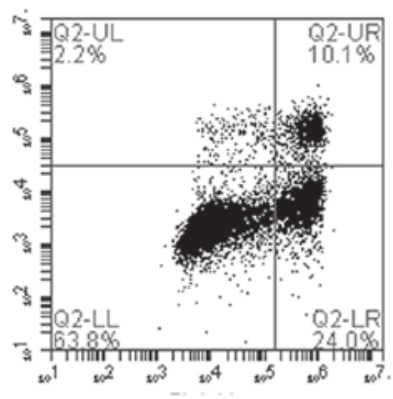

Srsf7-siRNA-2

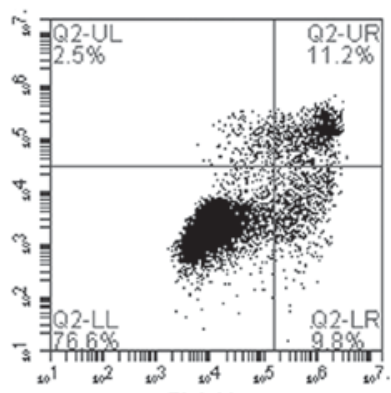

Annexin V

B

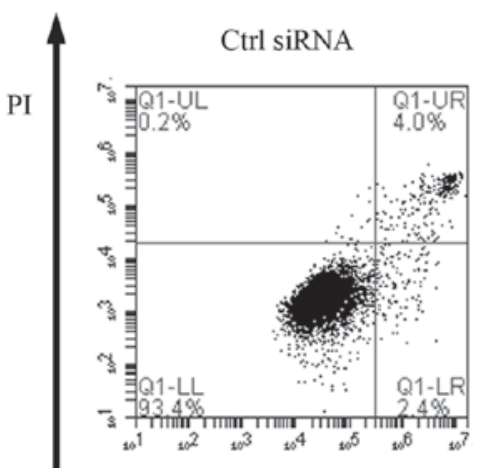

Srsf7-siRNA-1

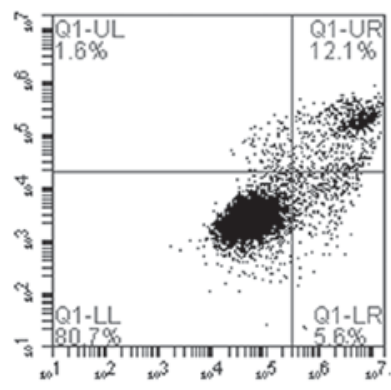

Srsf7-siRNA-2

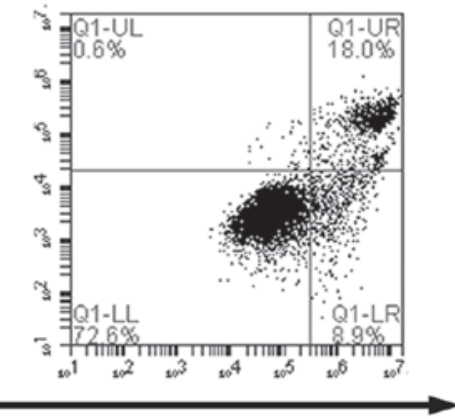

Annexin V

D

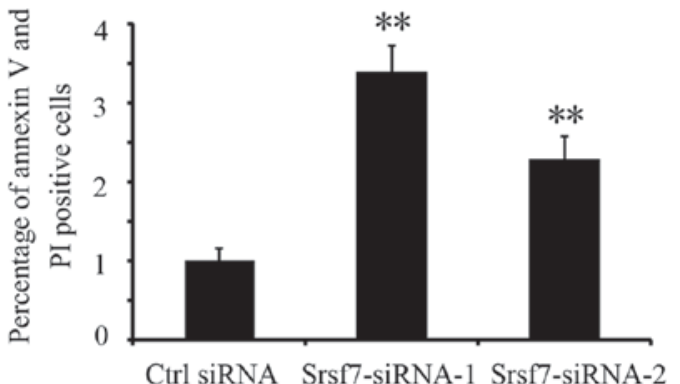

E

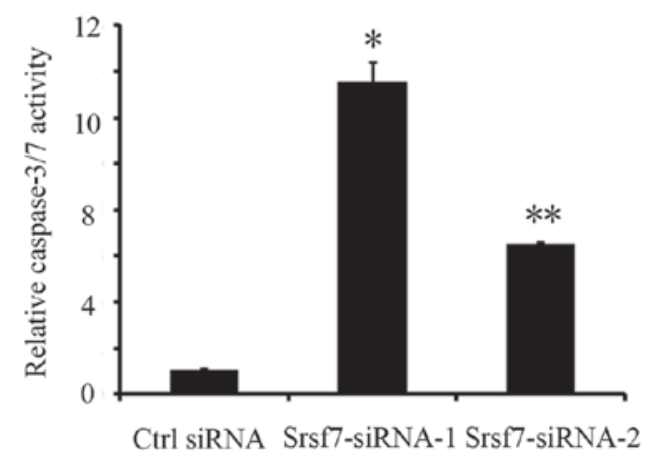

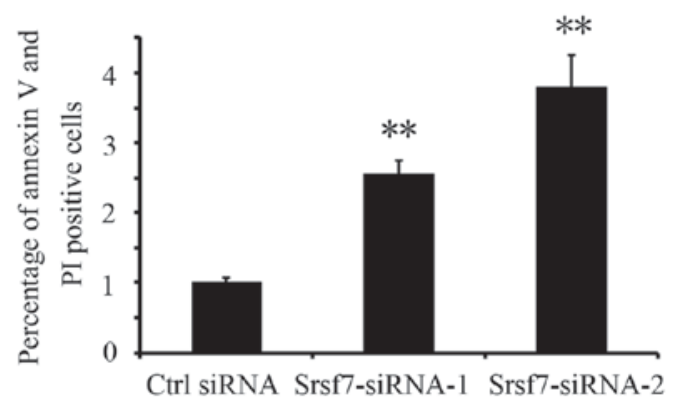

F

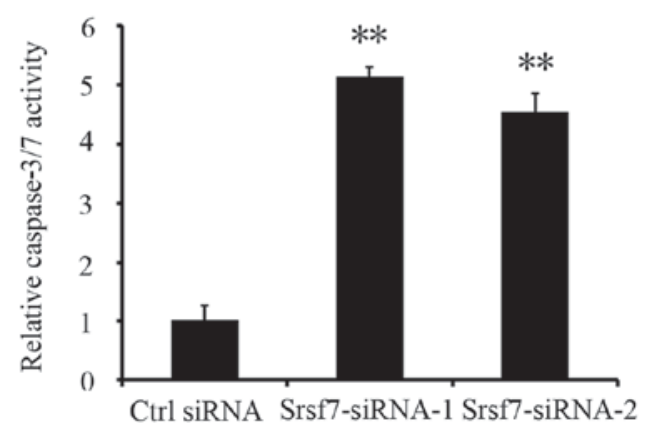

Figure 4. Downregulation of SRSF7 induces apoptosis in colon and lung cancer cells. (A) HCT116 and (B) A549 cells were transfected with control siRNA or SRSF7 siRNA for $72 \mathrm{~h}$ and subjected to an Annexin V-FITC and PI staining assay prior to flow cytometric analysis. Percentages of Annexin V-positive and PI-positive cells at $72 \mathrm{~h}$ post-siRNA transfection of (C) HCT116 and (D) A549 cells. Each bar represents the relative percentage of apoptotic cells compared with the control group. Error bars represent the standard deviation. ${ }^{* *} \mathrm{P}<0.01$, vs. Ctrl. (E) HCT116 and (F) A549 cells were transfected with control siRNA or SRSF7 siRNA for $72 \mathrm{~h}$ and assessed using an Apo-ONE Homogeneous Caspase-3/7 assay. Each bar represents the relative caspase-3/7 activity compared with the control group. Error bars represent the standard deviation. ${ }^{*} \mathrm{P}<0.05$ and ${ }^{* *} \mathrm{P}<0.01$, vs. Ctrl. SRSF7, serine/arginine-rich splicing factor 7; siRNA, small interfering RNA; Ctrl, control. 
A
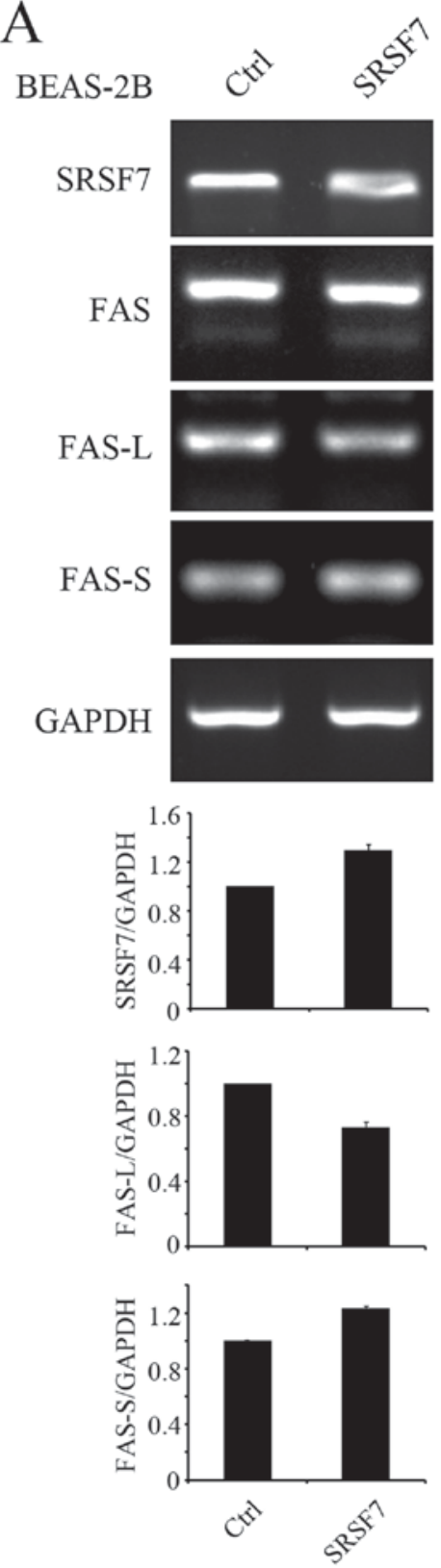
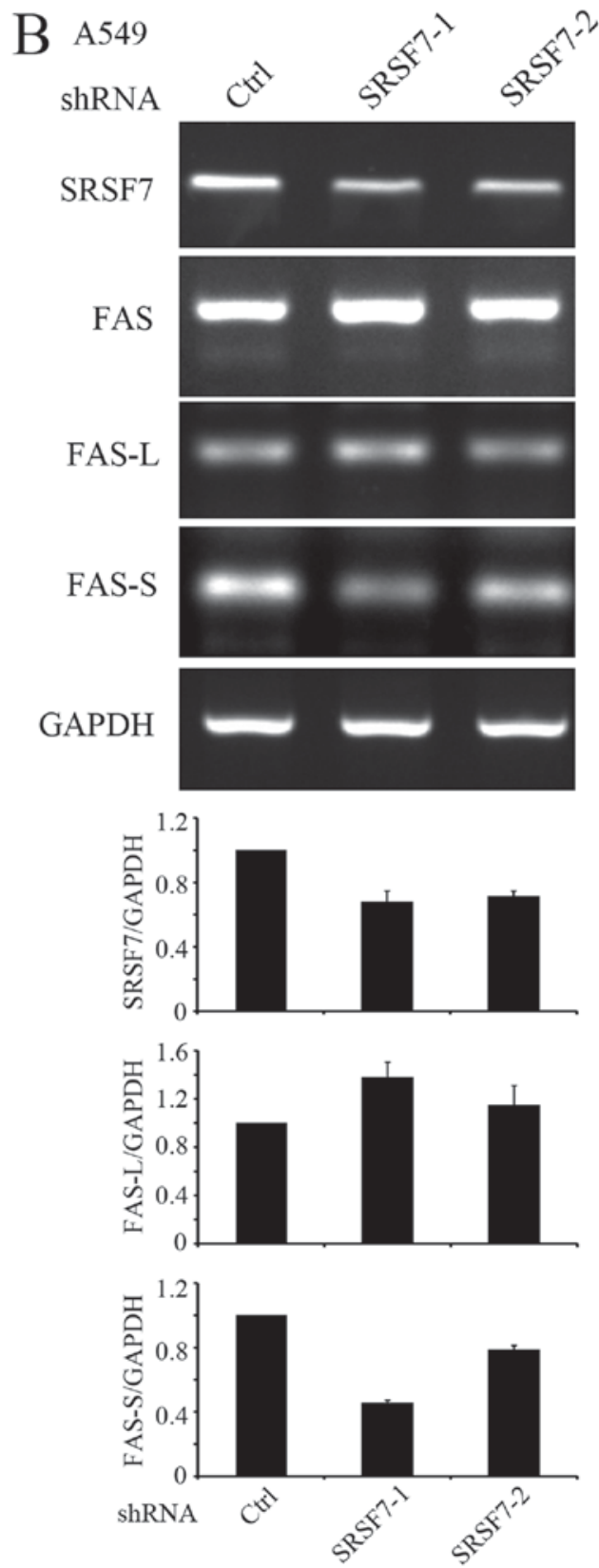

Figure 5. SRSF7 regulates the alternative splicing of Fas in human lung cells. (A) Total RNA was extracted from stable BEAS-2B cell lines expressing empty vector or SRSF7-overexpression vector. Following reverse transcription, cDNA was subjected to PCR, using primers to detect the alternative splicing of Fas. The relative expression levels of SRSF7, FAS-L and FAS-S against GAPDH were determined from three independent experiments. (B) Total RNA was extracted from A549 cell lines stably expressing empty vector or SRSF7-shRNA vectors. Following reverse transcription, cDNA was subjected to PCR, using primers that detected the alternative splicing of Fas. SRSF7, serine/arginine-rich splicing factor 7; FAS-L, Fas long isoform of exon 6 inclusion; FAS-S, Fas short isoform of exon 6 skipping; shRNA, short hairpin RNA; PCR, polymerase chain reaction; Ctrl, control.

in normal and cancerous human cell lines of the colon and lung. Western blot analysis revealed the upregulation of SRSF7 in the colon cancer cell lines (HCT116 and SW620) and lung cancer cell lines (A549 and H1299), compared with the normal cells (Fig. 2A and B). Among the colon and lung cancer cell lines, HCT116 and A549 cells were used in subsequent assays. To examine the function of SRSF7 in colon and lung cancer cell lines, downregulation experiments were performed to reduce the expression of SRSF7. The effect of SRSF7-inhibition on cell proliferation was then evaluated. The knockdown of SRSF7 was achieved in HCT116 and A549 cells using two different siRNAs, and its downregulation was confirmed by western blot analysis (Fig. 2C and D). The results of MTS assays demonstrated that the inhibition of SRSF7 markedly decreased the viabilities of the HCT116 and A549 cells, compared with those of the cells transfected with the control siRNA (Fig. 3A and B). These results suggested that SRSF7 is required for the proliferation of colon and lung cancer cells.

Downregulation of SRSF7 promotes apoptosis of HCT116 and A549 cells. To investigate the effect of the downregulation of SRSF7 on apoptosis, the rates of total apoptosis in the HCT116 and A549 cells were detected and quantified 
by flow cytometric analysis. The results showed that SRSF7 knockdown significantly increased the percentage of apoptotic cells $(\mathrm{P}<0.01)$ in the HCT116 and A549 cells, compared with the cells transfected with control siRNA (Fig. 4A-D). The induction of apoptosis in these cells was also confirmed by an increase in caspase 3/7 activity (Fig. 4E and F). These data indicated that SRSF7 is critical for the survival of colon and lung cancer cells.

SRSF7 regulates splicing of the apoptosis regulator Fas. To identify the alternative splicing events regulated by SRSF7, which may contribute to its effects on proliferation and apoptosis, the effects of the upregulation or downregulation of SRSF7 on the altered splicing events in cancer were examined. Non-malignant BEAS-2B lung epithelial cells were used to establish a stable SRSF7-overexpression cell line and A549 cells were used to establish a cell line with stable SRSF7 knockdown. In these stable cell lines, it was found that the splicing of Fas receptor was altered upon upregulation or downregulation of SRSF7. It has been reported that the alternative splicing of Fas exon 6 generates either a membrane-bound receptor that promotes apoptosis, or a soluble isoform that inhibits apoptosis (21). The results of the present study revealed that the upregulation of SRSF7 in BEAS-2B cells increased the skipping of Fas exon 6, whereas SRSF7 knockdown in A549 cells increased exon 6 inclusion (Fig. 5A and B). These results suggested that SRSF7 regulated the alternative splicing of Fas and promoted production of the more soluble, pro-survival variant.

\section{Discussion}

Colon and lung cancer are the leading causes of cancer-associated mortality worldwide (27). In particular, lung cancer has been associated with the highest rates of incidence in the last two decades in China (28). In the present study, the data indicated that SRSF7 was frequently upregulated in clinical colon and lung samples. It remains to be elucidated whether this upregulation involves mutational, transcriptional or epigenetic mechanisms. It was hypothesized that SRSF7 may act as a proto-oncogene in colon and lung cancer. However, the overexpression of SRSF7 in BEAS-2B cells cannot induce tumor growth in nude mice (data not shown). Therefore, the effects of SRSF7 in tumorigenesis may be dependent on other tumor-related genes.

To further investigate the function of SRSF7 in colon and lung cancer, the expression of SRSF7 was measured in colon and lung cancer cell lines. This indicated that the expression of SRSF7 was elevated in the HCT116 colon cancer cell line and A549 lung cancer cell line. The expression of SRSF7 was subsequently downregulated in these cell lines by siRNA, in order to examine the effect of SRSF7 on cell proliferation and apoptosis. It was observed that SRSF7 knockdown inhibited proliferation and promoted apoptosis of HCT116 and A549 cells. Similarly, Saijo et al (30) reported that SRSF7 knockdown induced G1 cell cycle arrest in HCT116 cells. These results indicate that SRSF7 exerts a crucial effect on the growth of colon and lung cancer cells.

Alterations in the alternative splicing of Fas have been shown to be an important activity of Fas in regulating cell apoptosis. Its transmembrane proapoptotic isoform, a soluble prosurvival isoform of Fas, can be expressed due to the skipping of exon 6 .
In the colon and lung cancer cells examined in the present study, the overexpression of SRSF7 promoted Fas exon 6 skipping and activated cell proliferation, whereas SRSF7 knockdown promoted Fas exon 6 inclusion and induced cell apoptosis. Therefore, SRSF7 may be involved in the growth of cancer cells by regulating the alternative splicing of Fas receptor pre-mRNA in cancer cells. Other downstream targets of SRSF7 in cancer cells require investigation in the future.

In conclusion, the findings of the present study suggested that SRSF7 knockdown inhibited the growth and promoted the apoptosis of colon and lung cancer cells by controlling apoptosis-related splicing events. Therefore, SRSF7 may be a potential therapeutic target for the treatment of colon and lung cancer. The specific molecular mechanisms underlying this activity of SRSF7 in cancer require further investigation.

\section{Acknowledgements}

The authors would like to thank Professor Wei Wu (School of Life Sciences, Tsinghua University, Beijing, China) for identifying an association between SRSF7 and cancer in his laboratory and Professor Xingfeng Li (College of Bioscience and Bioengineering, Hebei University of Science and Technology, Shijiazhuang, China) for providing the equipment.

\section{Funding}

This study was supported by grants awarded to $\mathrm{Dr} \mathrm{Yu} \mathrm{Fu}$ from the National Natural Science Foundation of China (grant no. 81402305), the Natural Science Foundation of Hebei Province (grant no. H2015208141), the Science and Technology Research Program for Colleges and Universities in Hebei Province (grant no. BJ2016024) and the Doctoral Scientific Research Foundation of Hebei University of Science and Technology (grant no. QD201411).

\section{Availability of data and materials}

The datasets used and analyzed during the current study are available from the corresponding author on reasonable request.

\section{Authors' contributions}

YF and YW designed the study and performed experiments, and YF wrote the manuscript.

\section{Ethics approval and consent to participate}

Not applicable.

\section{Consent for publication}

Not applicable.

\section{Competing interests}

The authors declare that they have no competing interests. 


\section{References}

1. Howard JM and Sanford JR: The RNAissance family: SR proteins as multifaceted regulators of gene expression. Wiley Interdiscip Rev RNA 6: 93-110, 2015.

2. Manley JL and Krainer AR: A rational nomenclature for serine/arginine-rich protein splicing factors (SR proteins). Genes Dev 24: 1073-1074, 2010.

3. Zhou Z and Fu XD: Regulation of splicing by SR proteins and SR protein-specific kinases. Chromosoma 122: 191-207, 2013

4. Sahebi M, Hanafi MM, van Wijnen AJ, Azizi P, Abiri R, Ashkani S, and Taheri S: Towards understanding pre-mRNA splicing mechanisms and the role of SR proteins. Gene 587: 107-119, 2016.

5. Twyffels L, Gueydan C and Kruys V: Shuttling SR proteins: More than splicing factors. FEBS J 278: 3246-3255, 2011.

6. Anczukow $\mathrm{O}$ and Krainer AR: Splicing-factor alterations in cancers. RNA 22: 1285-1301, 2016.

7. Shilo A, Siegfried Z and Karni R: The role of splicing factors in deregulation of alternative splicing during oncogenesis and tumor progression. Mol Cell Oncol 2: e970955, 2014.

8. Das S and Krainer AR: Emerging functions of SRSF1, splicing factor and oncoprotein, in RNA metabolism and cancer. Mol Cancer Res 12: 1195-1204, 2014.

9. Cavaloc Y, Popielarz M, Fuchs JP, Gattoni R and Stevenin J: Characterization and cloning of the human splicing factor 9G8 A novel $35 \mathrm{kDa}$ factor of the serine/arginine protein family. EMBO 13: 2639-2649, 1994.

10. Popielarz M, Cavaloc Y, Mattei MG, Gattoni R and Stevenin J: The gene encoding human splicing factor 9G8. Structure, chromosomal localization, and expression of alternatively processed transcripts. J Biol Chem 270: 17830-17835, 1995.

11. Galiana-Arnoux D, Lejeune F, Gesnel MC, Stevenin J, Breathnach R and Del Gatto-Konczak F: The CD44 alternative v9 exon contains a splicing enhancer responsive to the SR proteins 9G8, ASF/SF2, and SRp20. J Biol Chem 278: 32943-32953, 2003.

12. ten Dam GB, Zilch CF, Wallace D, Wieringa B, Beverley PC, Poels LG and Screaton GR: Regulation of alternative splicing of CD45 by antagonistic effects of SR protein splicing factors. J Immunol 164: 5287-5295, 2000.

13. Raponi M, Kralovicova J, Copson E, Divina P, Eccles D, Johnson P, Baralle D and Vorechovsky I: Prediction of single-nucleotide substitutions that result in exon skipping: Identification of a splicing silencer in BRCA1 exon 6. Hum Mutat 32: 436-444, 2011.

14. Gao L, Wang J, Wang Y and Andreadis A: SR protein 9G8 modulates splicing of tau exon 10 via its proximal downstream intron, a clustering region for frontotemporal dementia mutations. Mol Cell Neurosci 34: 48-58, 2007.

15. Huang Y and Steitz JA: Splicing factors SRp20 and 9G8 promote the nucleocytoplasmic export of mRNA. Mol Cell 7: 899-905, 2001.

16. Swartz JE, Bor YC, Misawa Y, Rekosh D and Hammarskjold ML: The shuttling SR protein 9G8 plays a role in translation of unspliced mRNA containing a constitutive transport element. J Biol Chem 282: 19844-19853, 2007.
17. Hatakeyama S, Sugihara K, Nakayama J, Akama TO, Wong SM, Kawashima H, Zhang J, Smith DF, Ohyama C, Fukuda M and Fukuda MN: Identification of mRNA splicing factors as the endothelial receptor for carbohydrate-dependent lung colonization of cancer cells. Proc Natl Acad Sci USA 106: 3095-3100, 2009.

18. Kim HR, Lee GO, Choi KH, Kim DK, Ryu JS, Hwang KE, Na KJ, Choi C, Kuh JH, Chung MJ, et al: SRSF5: A novel marker for small-cell lung cancer and pleural metastatic cancer. Lung Cancer 99: 57-65, 2016.

19. Bouillet P and O'Reilly LA: CD95, BIM and T cell homeostasis. Nat Rev Immunol 9: 514-519, 2009.

20. Villa-Morales $\mathbf{M}$ and Fernandez-Piqueras J: Targeting the Fas/FasL signaling pathway in cancer therapy. Exp Opin Ther Targets 16: 85-101, 2012.

21. David CJ and Manley JL: Alternative pre-mRNA splicing regulation in cancer: Pathways and programs unhinged. Genes Dev 24: 2343-2364, 2010

22. Cheng J, Zhou T, Liu C, Shapiro JP, Brauer MJ, Kiefer MC, Barr PJ and Mountz JD: Protection from Fas-mediated apoptosis by a soluble form of the Fas molecule. Science 263: 1759-1762, 1994.

23. Kondera-Anasz Z, Mielczarek-Palacz A and Sikora J: Soluble Fas receptor and soluble Fas ligand in the serum of women with uterine tumors. Apoptosis 10: 1143-1149, 2005.

24. Sheen-Chen SM, Chen HS, Eng HL and Chen WJ: Circulating soluble Fas in patients with breast cancer. World J Surg 27: 10-13, 2003.

25. Liu JH, Wei S, Lamy T, Li Y, Epling-Burnette PK, Djeu JY and Loughran TP Jr: Blockade of Fas-dependent apoptosis by soluble Fas in LGL leukemia. Blood 100: 1449-1453, 2002.

26. Tejedor JR, Papasaikas P and Valcarcel J: Genome-wide identification of Fas/CD95 alternative splicing regulators reveals links with iron homeostasis. Mol Cell 57: 23-38, 2015.

27. Siegel RL, Miller KD and Jemal A: Cancer statistics, 2015. CA Cancer J Clin 65: 5-29, 2015.

28. Chen W, Zheng R, Baade PD, Zhang S, Zeng H, Bray F, Jemal A, Yu XQ and He J: Cancer statistics in China, 2015. CA Cancer J Clin 66: 115-132, 2016.

29. Fu Y, Huang B, Shi Z, Han J, Wang Y, Huangfu J and Wu W: SRSF1 and SRSF9 RNA binding proteins promote Wnt signalling-mediated tumorigenesis by enhancing beta-catenin biosynthesis. EMBO Mol Med 5: 737-750, 2013.

30. Saijo S, Kuwano Y, Masuda K, Nishikawa T, Rokutan K and Nishida K: Serine/arginine-rich splicing factor 7 regulates p21-dependent growth arrest in colon cancer cells. J Med Invest 63: 219-226, 2016.

This work is licensed under a Creative Commons Attribution-NonCommercial-NoDerivatives 4.0 International (CC BY-NC-ND 4.0) License. 